Pacific Journal of Mathematics

ON THE CHARACTERIZATIONS OF THE BREAKDOWN
POINTS OF QUASILINEAR WAVE EQUATIONS 


\section{ON THE CHARACTERIZATIONS OF THE BREAKDOWN POINTS OF QUASILINEAR WAVE EQUATIONS}

\section{Peter H. Chang}

We consider the mixed initial and boundary value problem of the quasilinear wave equation:

$$
\begin{gathered}
u_{t}-v_{x}=0, \\
v_{t}-Q^{2}(u) u_{x}=0 ; \\
u(x, 0)=0, v(x, 0)=v_{0}(x), 0 \leqq x \leqq 1, \\
v(0, t)=v(1, t)=0, t \geqq 0 .
\end{gathered}
$$

In general the solution of the system (1), (2) eventually breaks down in the sense that some of its first derivatives become unbounded at a finite time. It is shown that there are only finitely many breakdown points and that at each of them there originates one or two shock curves.

The fact that solutions of (1), (2) eventually break down has been derived by many authors, e.g., see [12], [9], [5], [10], [6], [7], and [1]. It should be pointed out however, that even though a solution break down in finite time it can be extended to large $t$ as a weak solution. See e.g., [3], [4], and [11].

We define $r=v+M(u)$ and $s=v-M(u)$, where $M(\xi)=$ $\int_{0}^{\xi} Q(\zeta) d \zeta$. Then $r$ and $s$ are Riemann invariants of $(1)$. Let $q(\eta)=$ $Q\left(M^{-1}(\eta / 2)\right)$. After transforming (1) to equations with $r$ and $s$ as dependent variables we apply a hodograph transformation to invert the resulting equations to

$$
\begin{aligned}
& x_{r}-q(r-s) t_{r}=0, \\
& x_{s}+q(r-s) t_{s}=0 .
\end{aligned}
$$

Eliminating $x$ in (3) gives

$$
t_{r s}=\rho(r-s)\left(t_{r}-t_{s}\right)
$$

where $\rho(\eta)=Q^{\prime}\left(M^{-1}(\eta / 2)\right) / 4 q^{2}(\eta)$.

We assume that

$$
Q(\xi) \text { is a positive analytic function over }(-\infty, \infty) \text {; }
$$

$$
v_{0}(x)=\left.f\right|_{[0,1]}(x) \text { is concave over }[0,1] \text {, where } f(x) \text { is an odd }
$$
periodic analytic function over $(-\infty, \infty)$ with period 2 .

Let $\quad a=\max _{0 \leqq x \leqq 1} f(x)=f(b)(0<b<1), \Omega=\{(r, s):|r| \leqq a ;|s| \leqq a\}$, $\Omega_{i}=\left\{(r, s) \in \Omega:(-1)^{i-1}(r-s) \geqq 0\right\}(i=1,2), a_{1}=M(\infty)$ and $a_{2}=$ $-M(-\infty)$. We assume further that 
(7) $\partial^{i} \rho / \partial r^{i}(r-s)=(-1)^{i} \partial^{i} \rho / \partial s^{i}(r-s)<0$ for $i \geqq 0$ and $(r, s) \in \Omega$;

$$
a<\min \left(a_{1}, a_{2}\right) \text {. }
$$

We refer the part in (7) with $i=0$ as (7) $(i=0)$ and that in (7) with $i>0$ as $(7)(i>0)$. We refer a breakdown point as a b.d.p. We call a shock curve of the equations (1) propagating to the left an 1-shock and that propagating to the right a 2-shock.

In this paper applying a method developed in [2] we prove that the system (1), (2) has only finitely many b.d.p.'s. Each b.d.p. $P_{0}$ can be characterized as being of one of the following two kinds:

I. There is exactly one shock curve originating at $P_{0}$, which is either an 1-shock or a 2 -shock.

II. There are exactly two shock curves originating at $P_{0}$; one is an 1-shock and another a 2-shock.

The methods used in this paper are applicable to some other problems of (1) (see Remark 1). The condition (7) is satisfied by the system which governs the motion of an isentropic polytropic gas (see Remark 2). I am grateful to Professor H. Weinberger and to the referee for several helpful comments.

The condition (7) $(i=0)$ is the genuine nonlinearity condition of a hyperbolic 2-conservation law. Without imposing $(7)(i>0)$ we find two other possible characterizations of a b.d.p. $P_{0}$.

III. There is a (infinite) sequence of shocks originating at a sequence of points $\left\{P_{k}\right\}$ convergent to $P_{0}$. Moreover, the set $\left\{P_{k}\right\}$ is the only set with the above property in a neighborhood of $P_{0}$ and $\left\{P_{k}\right\}$ contains no subsequence convergent to a point other than $P_{0}$.

IV. There is a sequence of points $\left\{P_{k}\right\}$ convergent to $P_{0}$ such that for each $k$ there is a sequence of shocks originating at a sequence of points convergent to $P_{k}$.

The condition (8) is the first case of MacCamy and Mizel [10]. The second and third cases are $\min \left(a_{1}, a_{2}\right) \leqq a \leqq \max \left(a_{1}, a_{2}\right)$ and $a>\max \left(a_{1}, a_{2}\right)$. Under (7) $a_{2}=\infty$ and the third case does not occur. As will be seen in Section 2 a local solution of (1), (2) can be constructed and extended from some solutions $X(R)=(x(r, s), t(r, s))$ of (3) over $\Omega$. The second case differs from (8) in the shapes of the images $X(\Omega)$ in the $(x, t)$ plane. For this case applying the methods used in this paper we obtain the same kinds of characterizations of the b.d.p.'s. 
From now on in this section and $\S 2$ the results are derived without assuming (7). The following lemmas are Lemmas 1, 2, and 3 of [1].

Lemma 1. Suppose $X(R)$ is a solution of (3) in an open region $W$ with the property that any two points in $W$ can be connected by one of the following: (i) a horizontal segment or a vertical segment in $W$, (ii) one or two line segments in $W$ of positive slopes, (iii) one or two line segments in $W$ of negative slopes, and if $t_{r} \cdot t_{s} \neq 0$ in $W$, then $X(R)$ is a homeomorphism on $W$.

Lemma 2. Suppose $X(R)$ is a solution of (3). If $W$ is an open region in which $X(R)$ is one-to-one with nonvanishing Jacobian $\operatorname{det} \nabla_{R} X=2 q t_{r} t_{s}$, then

$$
U(X)=\left(M^{-1}\left(\frac{r(x, t)-s(x, t)}{2}\right), \frac{r(x, t)+s(x, t)}{2}\right)
$$

is a solution of (1) on $X(W)$.

Lemma 3. If $X$ satisfies the assumptions of Lemma 2 and is continuous on $\bar{W}$, and if $t_{r} \rightarrow 0$ or $t_{s} \rightarrow 0$ as $R \rightarrow R_{0} \in \partial W$, then $\left|u_{x}(X(R))\right| \rightarrow \infty$ or $\left|v_{x}(X(R))\right| \rightarrow \infty$ as $R \rightarrow R_{0}$.

2. The existence of a local solution. The equation (4) can be written

$$
\begin{aligned}
& \left(\sqrt{q} t_{r}\right)_{s}=q^{*}(r-s) t_{s}, \text { or } \\
& \left(\sqrt{q} t_{s}\right)_{r}=-q^{*}(r-s) t_{r}
\end{aligned}
$$

where $q^{*}(\eta)=-Q^{\prime}\left(M^{-1}(\eta / 2)\right) / 4 q^{3 / 2}(\eta)$. Integrating (10) and (11) with the diagonal $r=s$ of $\Omega$ as initial curve and applying successive approximations we have

Lemma 4. Suppose $t(r, s)$ is a solution of (4) over $\Omega$. For $i=1$ or 2 , if $(-1)^{i} Q^{\prime}\left(M^{-1}((r-s) / 2)\right)>0$ in $\Omega_{i}$ and if $(-1)^{i} t_{r}<0$ and $(-1)^{i} t_{s}>0$ along the diagonal $r=s$ of $\Omega$, then $(-1)^{2} t_{r}<0$ and $(-1)^{i} t_{s}>0$ in $\Omega_{i}$.

Assuming $Q^{\prime}(\xi)>0$ for $\xi<0$ and $Q^{\prime}(\xi)<0$ for $\xi>0$, MacCamy and Mizel [10] construct the local solution of (1), (2) as follows. Let $f_{1}(x)$ be the portion of $f(x)$ over $[-b, b]$ and $f_{2}(x)$ that over $[b, 2-b]$. Solving (3) with the initial conditions

$$
x_{i}^{0}(r, r)=f_{i}^{-1}(r), t_{i}^{0}(r, r)=0
$$


one obtains a solution $X_{i}^{\wedge}(R)=\left(x_{i}^{0}(r, s), t_{i}^{\imath}(r, s)\right)$ over $\Omega_{i}(i=1,2)$. Differentiating (12) with respect to $r$ and using (3) we find

$$
(-1)^{i} t_{i r}^{0}(r, r)=(-1)^{i+1} t_{i s}^{0}(r, r)=(-1)^{i} f_{i}^{-1}(r) / 2 q(0)<0 \quad(i=1,2) .
$$

It follows from Lemma 4 that

$$
(-1)^{i} t_{i r}^{0}<0 \text { and }(-1)^{i} t_{i s}^{0}>0 \text { in } \Omega_{i}(i=1,2) \text {. }
$$

By (6) and the uniqueness theorem of the initial value problem of (3) we can derive that

$$
X_{i}^{0}(R)=\left(1+(-1)^{i}-x_{i}^{0}(-s,-r), t_{i}^{0}(-s,-r)\right)(i=1,2) .
$$

Solving (3) with the characteristic initial conditions

$$
X^{1}(a, s)=X_{1}^{0}(a, s), X^{1}(r, a)=X_{2}^{0}(r, a)
$$

one obtains a solution $X^{1}(R)=\left(x^{1}(r, s), t^{1}(r, s)\right)$ over $\Omega$. Let $\left\{r=r_{0}\right\}$ and $\left\{s=s_{0}\right\}$ denote the line segments $r=r_{0}$ and $s=s_{0}$ in $\Omega$. By (16) and (14) there exists a neighborhood $Z$ of $\{r=a\} \cup\{s=a\}$ in $\Omega$ such that

$$
t_{r}^{1}<0 \text { and } t_{s}^{1}<0 \text { in } Z \text {. }
$$

Let $D_{i}^{0}=X_{i}^{0}\left(\Omega_{i}\right)(i=1,2)$. By (14), (17), Lemmas 1 and 2 the function defined by $U(X)=U\left(X_{i}^{0}\right)$ for $X \in D_{i}^{\circ}(i=1,2)$ and $U(X)=U\left(X^{1}\right)$ for $X \in X^{1}(Z)$ as in (9) is a solution in $D_{1}^{0} \cup X^{1}(Z) \cup D_{2}^{0}$ of (1) with initial conditions $u(x, 0)=0, v(x, 0)=f(x)$ for $-b \leqq x \leqq 2-b$. By (15) the function $U(X)$ satisfies $U(X)=\left(u\left(1+(-1)^{i}-x, t\right),-v(1+\right.$ $\left.\left.(-1)^{i}-x, t\right)\right)$ for $X \in D_{i}^{0}(i=1,2)$. Thus $U(X)$ is a local solution of (1), (2). As we stated in Remark 2 of [1], without assuming any condition on the signs of $Q^{\prime}$, applying (13) and Lemma 1 we can also construct such a local solution.

From now on in this section we impose no condition on the signs of $Q^{\prime}$. Constructing $X^{1}(R)$ as previously described we can solve a series of characteristic initial value problems as described in $\S 3$ of [10] and construct the functions $X_{i}^{2 k}, X_{i}^{2 k+1}$, and $X^{2 k+1}$ over $\Omega$. For later reference we list the characteristic initial conditions for the functions $X_{i}^{1}, X_{i}^{\imath}(i=1,2)$, and $X^{3}$ as follows:

$$
\begin{aligned}
& X_{i}^{1}(-a, s)=\left(x_{2}^{0}(-a, s)-1+(-1)^{i}, t_{2}^{0}(-a, s)\right), \\
& X_{i}^{1}(r,-a)=\left(x_{1}^{0}(r,-a)+1+(-1)^{i}, t_{1}^{0}(r,-a)\right) ; \\
& X_{1}^{2}(a, s)=X_{1}^{1}(a, s), X_{1}^{2}(r,-a)=X^{1}(r,-a) ; \\
& X_{2}^{2}(-a, s)=X^{1}(-a, s), X_{2}^{2}(r, a)=X_{2}^{1}(r, a) ; \\
& X^{3}(-a, s)=X_{1}^{2}(-a, s), X^{3}(r,-a)=X_{2}^{2}(r,-a) .
\end{aligned}
$$

Let $D^{2 k+1}=X^{2 k+1}(\Omega), D_{i}^{2 k+1}=X_{i}^{2 k+1}(\Omega)$, and $D_{i}^{2 k}=X_{i}^{2 k}(\Omega)$ (see Fig. 1). 


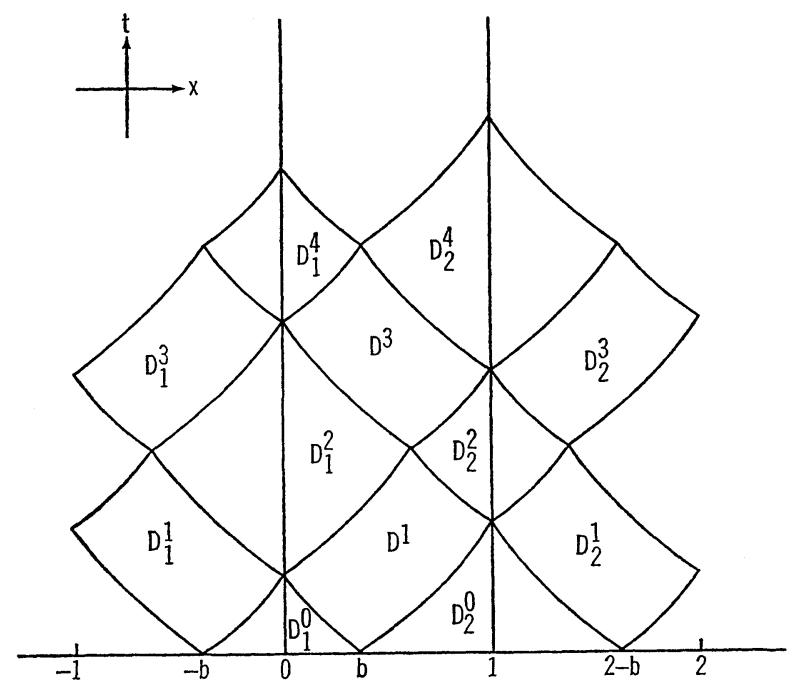

Figure 1

By (15), (16), (18), and the uniqueness theorem of the characteristic initial value problem of (3) we can derive that

$$
\begin{aligned}
& X^{2 k+1}(R)=\left(1+(-1)^{i}-x_{i}^{2 k+1}(-s,-r), t_{i}^{2 k+1}(-s,-r)\right), \\
& X_{i}^{2 k+2}(R)=\left(1+(-1)^{i}-x_{i}^{2 k+2}(-s,-r), t_{i}^{2 k+2}(-s,-r)\right)
\end{aligned}
$$

for $k \geqq 0$ and $i=1,2$.

Let $\Psi(R)=t^{1}(R)+t^{1}(-s,-r)-t_{1}^{0}(R)-t_{2}^{0}(R)$. The following is Lemma 5 of [1].

LEMMA 5 . For $k \geqq 0 \quad$ and $i=1,2, t^{4 k+1}(R)=2 k \Psi(R)+t^{1}(R)$, $t_{i}^{4 k+2}(R)=(2 k+1) \Psi(R)+t_{i-(-1)}^{0}(R), t^{4 k+3}(R)=(2 k+1) \Psi(R)+t^{1}(-s,-r)$, $t_{i}^{4 k+4}(R)=(2 k+2) \Psi(R)+t_{i}^{0}(R)$.

Let $\Omega$ denote the interior of $\Omega$ and $V$ the set of four vertices $\{(a, a),(-a, a),(-a,-a),(a,-a)\}$ of $\Omega$.

Lemma 6 . For $k \geqq 0$ and $i=1,2$ the following hold.

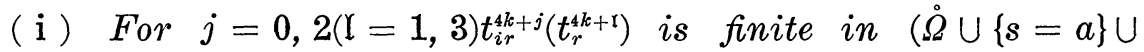

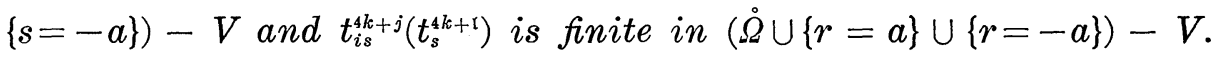

(ii) Along $\{r=a\} \cup\{r=-a\}$

$$
t_{1 r}^{4 k}=-t_{2 r}^{4 k}=-t_{r}^{4 k+1}=-t_{1 r}^{4 k+2}=t_{2 r}^{4 k+2}=t_{r}^{4 k+3}=\infty .
$$

(iii) Along $\{s=a\} \cup\{s=-a\}$

$$
t_{1 s}^{4 k}=-t_{2 s}^{4 k}=t_{s}^{4 k+1}=-t_{18}^{4 k+2}=t_{2 s}^{4 k+2}=-t_{s}^{4 k+3}=-\infty .
$$

The parts with $k=0, j=0$, and $\mathfrak{l}=1$ of Lemma $6(\mathrm{i})$ and those 
with superscripts 0,1 of Lemma 6 (ii), (iii) are Lemmas 6 and 7 of [1]. By Lemma 8 of [1] the function $\Psi$ has finite first-order partial derivatives in $\Omega$. From Lemma 5 the other parts of Lemma 6 follow.

\section{The zero curves of $t_{2 r}^{0}$ and of $t_{2 s}^{0}$. We now assume (7).}

Lemma 7. Let $t(R)$ denote either $t_{i}^{2 k}(R)$ or $t^{2 k+1}(R)(k \geqq 0 ; i=1$, 2). If $C_{r}\left(C_{s}\right)$ is a zero curve of $t_{r}\left(t_{s}\right)$, then $C_{r}\left(C_{s}\right)$ contains no horizontal line segment or vertical line segment.

Proof. Since $Q(\xi)$ and $f(x)$ are analytic, the function $t(R)$ is analytic. For a fixed $s_{0}, t_{r}\left(r, s_{0}\right)$ is analytic in $r$. Now a real analytic function of one variable either is a constant function or does not take a constant value on any set with an accumulation point. It follows from Lemma 6 (ii) that $t_{r}\left(r, s_{0}\right)$ does not vanish on a closed interval. Hence $C_{r}$ contains no horizontal segment. Similarly $C_{s}$ contains no vertical segment. By (10) and (11) we then derive that $C_{r}$ contains no vertical segment and that $C_{s}$ contains no horizontal segment.

\section{Applying (10) and (11) we can prove}

LEMMA 8. Let $t(R)$ denote either $t_{i}^{2 k}(R)$ or $t^{2 k+1}(R)(k \geqq 0 ; i=$ 1,2). If $t_{r}\left(r_{1}, s_{1}\right)=t_{r}\left(r_{1}, s_{2}\right)=0$ along $\left\{r=r_{1}\right\}$, then $t_{s}\left(r_{1}, s_{0}\right)=0$ for some $s_{0}$ between $s_{1}$ and $s_{2}$. If $t_{s}\left(r_{3}, s_{3}\right)=t_{s}\left(r_{4}, s_{3}\right)=0$ along $\left\{s=s_{3}\right\}$, then $t_{r}\left(r_{0}, s_{3}\right)=0$ for some $r_{0}$ between $r_{3}$ and $r_{4}$.

Under (7) by Theorem 4(i) of [1] the solution $U$ of (1), (2) breaks down. By Lemma $4, t_{1 r}^{0}>0$ and $t_{1 s}^{0}<0$ in $\Omega_{1}$. It follows from Lemmas 2 and 3 that $U$ does not break down in $D_{1}^{0}$. Now $Q^{\prime}\left(M^{-1}((r-s) / 2)\right)<0$ in $\Omega_{2}$. The method of successive approximations used in showing Lemma 4 is not applicable to show that $t_{2 r}^{0}<0$ and $t_{2 s}^{0}>0$ in $\Omega_{2}$. In this section we assume that $t_{2 r}^{0} \cdot t_{2 s}^{0}$ vanishes somewhere in $\Omega_{2}$. That is, $U$ breaks down in $D_{2}^{0}$. By (15)

$$
t_{2 r}^{0}(r, s)=-t_{2 s}^{0}(-s,-r) \text { for }(r, s) \in \Omega_{2} .
$$

Then both $t_{2 r}^{0}$ and $t_{2 s}^{0}$ vanish somewhere in $\Omega_{2}$.

From now on in this section let $X(R)=(x(R), t(R))$ denote $X_{2}^{0}(R)$. By (13) $t_{r}(r, r)=-t_{s}(r, r)<0$. Let $F$ be the family of subsets $S$ of $\Omega_{2}$ which satisfy: (i) $S$ is open and connected with respect to the induced Enclidean topology on $\Omega_{2}$, (ii) $S$ contains the diagonal $r=s$, (iii) $t_{r}<0$ and $t_{s}>0$ in $S$. The set $N=\bigcup_{S \in F} S$ is the largest member of $F ; N \varsubsetneqq \Omega_{2}$. Let $A_{r}=\left\{R \in \Omega_{2}: t_{r}(R)=0\right\}$ and $A_{s}=\{R \in$ 
$\left.\Omega_{2}: t_{s}(R)=0\right\}$

Lemma 9. (i) In $N, t_{r}<0$ and $t_{s}>0$. (ii) $\partial N \cong A_{r} \cup A_{s}$.

For a fixed number $r^{0}$ we denote the curve $t(r, s)=t\left(r^{0},-r^{0}\right)$ through the point $\left(r^{0},-r^{0}\right)$ by $C_{t\left\langle r^{n},-r^{0}\right\rangle}$. Let $\alpha=\inf \{r:-a<r<0$; $C_{t(r,-r)}$ lies in $\left.N\right\} \quad(-a<\alpha<0), T_{0}=t(\alpha,-\alpha)$, and $C_{0}=C_{T_{0}}$. Let $r=r_{0}(s), s^{*} \leqq s \leqq a$, describe $C_{0}$. From now on by an increasing (decreasing) function we mean a strictly increasing (decreasing) function. By a method similar to that used in showing Lemma 3.3 of [2] we can show

LEMMA 10. (i) The function $r_{0}(s)$ is increasing. (ii) $\partial N \cap C_{0} \neq \phi$. (iii) The interior of the region to the right of $C_{0}$ in $\Omega_{2}$ is contained in $N$.

We describe the $X$ images of the region $N$, of a curve $C_{t\left(r^{n},-r^{n}\right)}\left(r^{0}>\alpha\right)$, and of the curve $C_{0}$ in Fig. 2. By Lemma 9 and Lemma 10 (iii)

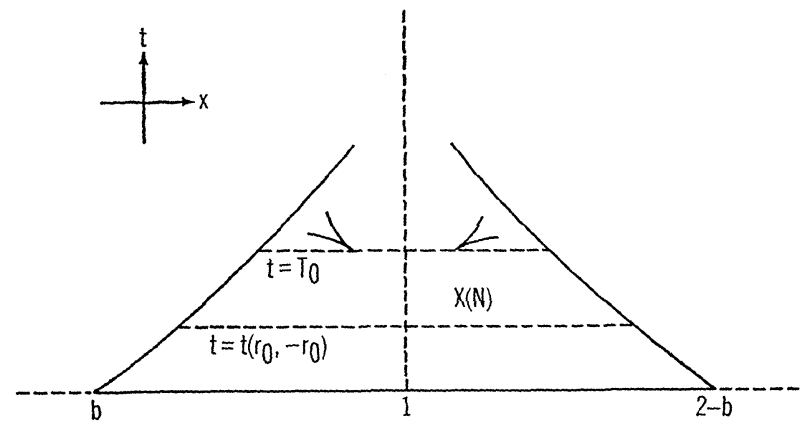

Figure 2

$$
\partial N \cap C_{0}=\left(A_{r} \cap C_{0}\right) \cup\left(A_{s} \cap C_{0}\right) .
$$

Lemma 11. The set $\partial N \cap C_{0}$ is countable.

Proof. For any arc $\gamma$ of $C_{0}$, applying Lemma 7, integrating (10) and (11) with $\gamma$ as initial curve, and applying successive approximations, we can prove that $A_{r} \cap \gamma \varsubsetneqq \gamma$ and $A_{s} \cap \gamma \varsubsetneqq \gamma$. Then the closed sets $\left\{s: s^{*} \leqq s \leqq a ; t_{r}\left(r_{0}(s), s\right)=0\right\}$ and $\left\{s: s^{*} \leqq s \leqq a ; t_{s}\left(r_{0}(s)\right.\right.$, $s)=0$ c contain no closed interval. Thus both of them are countable. It follows from (21) that $\partial N \cap C_{0}$ is countable.

We denote a zero curve $s=s(r)(r=r(s))$ of $\partial^{m} t / \partial r^{m}\left(\partial^{m} t / \partial s^{m}\right)$ in $\Omega_{2}$ by $C_{r^{m}}\left(C_{s^{m}}\right)(m \geqq 1)$ and $C_{r^{1}}\left(C_{s^{1}}\right)$ by $C_{r}\left(C_{s}\right)$. We call a number $x_{0}$ a 
relative maximum (minimum) point of a certain function $\phi(x)$ if $\phi(x)$ has a relative maximum (minimum) at $x_{0}$. We call $x_{0}$ a relative extreme point of $\phi(x)$ if it is either a relative maximum point or a relative minimum point of $\phi(x)$.

Definition 1. A point $R_{0}=\left(r_{0}, s_{0}\right)$ is a bending maximum point of $C_{r^{m}}\left(C_{s^{m}}\right)$ if the function $s=s(r)(r=r(s))$ describing $C_{r^{m}}\left(C_{s^{m}}\right)$ has exactly one relative extreme point $r_{0}\left(s_{0}\right)$ in a neighborhood of $r_{0}\left(s_{0}\right)$, which is a relative maximum point. We define the bending minimum point similarly. The point $R_{0}$ is a bending point of $C_{r^{m}}\left(C_{s} m\right)$ if it is either a bending maximum point or a bending minimum point of $C_{r^{m}}\left(C_{s^{m}}\right) . \quad A C_{r^{m}}\left(C_{s^{m}}\right)$ is of the first kind through $R_{0}$ if it contains only finitely many bending points in a neighborhood of $R_{0}$.

In considering $A_{r} \cap C_{0}$ and $A_{s} \cap C_{0}$, by (21), Lemma 10 (ii), and Lemma 6(ii), (iii), we have the following five cases:

Case 1. $\left(A_{r} \cap C_{0} \cap \stackrel{\circ}{2}_{2}\right)-A_{s} \neq \phi$.

Case 2. $\left(A_{r} \cap C_{0} \cap\{s=a\}\right)-\left(A_{s} \cup V\right) \neq \phi$.

Case 3. $\left(A_{s} \cap C_{0} \cap \stackrel{\circ}{\Omega}_{2}\right)-A_{r} \neq \phi$.

Case 4. $\left(A_{s} \cap C_{0} \cap\{r=-a\}\right)-\left(A_{r} \cup V\right) \neq \phi$.

Case 5. $A_{r} \cap A_{s} \cap C_{0} \cap \stackrel{\Omega}{\Omega}_{2} \neq \phi$.

We consider whether there exists a $C_{r}$ or a $C_{s}$ through each point in these intersections and whether these intersections contain only isolated points of $\partial N \cap C_{0}$.

For Case 1, given $R_{0} \in\left(A_{r} \cap C_{0} \cap \stackrel{\Omega}{2}_{2}\right)-A_{s}$, by a method similar to that used in Case 1 of $\S 3$ of [2], we can show that there is a unique $C_{r}$, which is of the first kind through $R_{0}$, with $R_{0}$ as a bending minimum point. We describe this $C_{r}$ in Fig. 3. By (21) the point $R_{0}$ is an isolated point of $\partial N \cap C_{0}$.

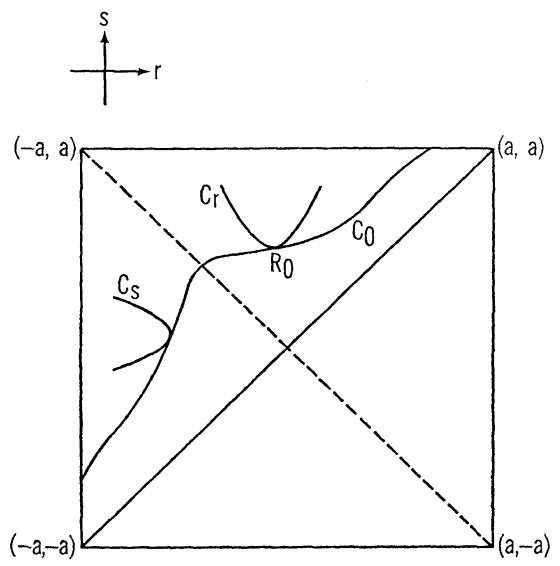

Figure 3 


\section{For Case 2 we prove}

Lemma 12. Given $R_{0}=\left(r_{0}, a\right) \in\left(A_{r} \cap C_{0} \cap\{s=a\}\right)-\left(A_{s} \cup V\right)$, the following hold.

(i) There is a deleted neighborhood of $R_{0}$ in $\Omega_{2}$ in which $t_{r}<0$.

(ii) There is a unique zero curve $C_{r}$ of $t_{r}^{1}$, which is of the first kind through $R_{0}$, with $R_{0}$ as a bending maximum point. Suppose $s=s(r)$ describes $C_{r}$. We also have that $t_{r}^{1}(r, s)<0(>0)$ for $s>s(r)$ $(s<s(r))$ and $\left|r-r_{0}\right|$ small.

(iii) The point $R_{0}$ is an isolated point of $\partial N \cap C_{0}$.

Proof. By Lemma 6(iii) there is a convex neighborhood $W$ of $R_{0}$ in $\Omega_{2}$ such that $t_{\mathrm{s}}>0$ in $W$. Suppose there is a zero curve $C_{r}$ of $t_{r}$ through $R_{0}$. By Lemma 8 it is the only such curve through $R_{0}$. By Lemma 10 (iii) $C_{r}$ lies to the left of $C_{0}$. In $W$ we choose a point $R^{0}=\left(r^{0}, s^{0}\right)$ in $\dot{\Omega}_{2}$ lying strictly between $C_{r}$ and $C_{0}$ such that the line segment $\left\{\left(r, s^{0}\right): r^{0} \leqq r \leqq r_{0}\left(s^{0}\right)\right\}$ lies in $N$. Then $t\left(R^{0}\right)>t\left(r_{0}\left(s^{0}\right), s^{0}\right)=T_{0}$. By (10) we can show, however, that the curve $t(r, s)=t\left(R^{0}\right)$ through $R^{0}$ meets $C_{0}$. This is a contradiction. Thus there is no zero curve of $t_{r}$ through $R_{0}$. This derives (i).

By (16) $t_{r}^{1}(r, a)=t_{r}(r, a)(-a \leqq r \leqq a)$. By (4), (7), and Lemma 6(iii) $t_{r s}^{1}<0$ in a neighborhood $W_{1}$ of $R_{0}$ in $\Omega_{2}$. Then $t_{r}^{1}\left(r_{0}, s\right)>0$ for small positive $a-s$. Since $t_{r}^{1}(r, a)<0$ for $r$ in a deleted neighborhood of $r_{0}$, we may choose $W_{1}$ sufficiently small such that $t_{r}^{1}<0$ along $\left(W_{1} \cap\{s=a\}\right)-\left\{R_{0}\right\}$. It follows from (10) that there is a unique zero curve $C_{r}$ of $t_{r}^{1}$ lying in $\stackrel{\circ}{W}_{1} \cup\left\{R_{0}\right\}$ through $R_{0}$.

We prove that $C_{r}$ is of the first kind through $R_{0}$.

Since $t_{r}^{1}(r, a)$ is analytic in $r$ at $r_{0}$, there is an even number $m$ such that

$$
\partial^{i} t_{r}^{1} / \partial r^{i}\left(R_{0}\right)=0(0 \leqq i \leqq m-1) \text { and } \delta=\partial^{m} t_{r}^{1} / \partial r^{m}\left(R_{0}\right)<0 .
$$

We claim that for $R$ in some neighborbood $W_{2}$ of $R_{0}$,

$$
\partial^{k} t_{s}^{1} / \partial r^{k}(R)<0 \quad(0 \leqq k \leqq m)
$$

For, by Lemma 6(iii) $t_{s}^{1}<0$ in a neighborhood of $R_{0}$. For $m \leqq 4$ or for $k \leqq 3$, by (4), (22), and (7) we can verify directly that (23) holds. For $m \geqq k \geqq 4$, by (4), (22), (7), and by induction we can prove that in a small neighborhood of $R_{0}$, the sign of the function $\partial^{k} t_{s}^{1} / \partial r^{k}$ is the same as that of the following function:

$$
-\partial^{k-1} \rho / \partial r^{k-1} t_{s}^{1}+\left(\begin{array}{l}
k-1 \\
k-2
\end{array}\right) \partial^{k-2} \rho / \partial r^{k-2} \rho t_{s}^{1}+\left(\begin{array}{l}
k-1 \\
k-3
\end{array}\right) \partial^{k-3} \rho / \partial r^{k-3}\left(\rho_{r}-\rho^{2}\right) t_{s}^{1}
$$




$$
\begin{aligned}
& +\sum_{i= \pm}^{k}\left(\begin{array}{l}
k-1 \\
k-i
\end{array}\right) \partial^{k-i} \rho / \partial r^{k-i}\left\{\partial^{i-2} \rho / \partial r^{i-2}\right. \\
& +\sum_{j=4}^{i}(-1)^{j-1}\left[\left(\begin{array}{l}
i-2 \\
i-j+1
\end{array}\right) \partial^{i-j+1} \rho / \partial r^{i-j+1} \rho^{j-3}\right. \\
& +\sum_{i=2}^{j-2}\left(\sum_{i_{2}=0}^{i-j}\left(\begin{array}{l}
i-2 \\
i_{2}
\end{array}\right) \partial^{i_{2}} \rho / \partial r^{i_{2}}\left(\sum_{i_{3}=0}^{i-j-i_{2}}\left(\begin{array}{l}
i-3-i_{2} \\
i_{3}
\end{array}\right) \partial^{i_{3}} \rho / \partial r^{i_{3}} \ldots\right.\right. \\
& \times\left(\sum_{i_{\mathfrak{l}}=0}^{i-j-i_{2}-\cdots-i_{\mathfrak{l}-1}}\left(\begin{array}{c}
i-\mathfrak{l}-i_{2}-\cdots-i_{\mathfrak{l}-1} \\
i_{\mathfrak{l}}
\end{array}\right) \partial^{i_{\mathfrak{\imath}}} \rho / \partial r^{i_{\mathfrak{l}}}\right. \\
& \times\left(\begin{array}{l}
i-\mathfrak{l}-1-i_{2}-\cdots-i_{\mathfrak{l}} \\
i-j+1-i_{2}-\cdots-i_{\mathfrak{l}}
\end{array}\right) \partial^{i-j+1-i_{2}-\cdots-i_{\mathfrak{l}}} \rho / \partial r^{2-j+1-i_{2}-\cdots-i_{1}} \\
& \left.\left.\left.\left.\times \rho^{j-1-2}\right) \cdots\right)\right]+(-1)^{i} \rho^{i-1}\right\} t_{s}^{1} .
\end{aligned}
$$

It follows from (7) that (23) holds.

By (22) we can choose $W_{2}$ sufficiently small in which $\partial^{m} t_{r}^{1} / \partial r^{m}<0$. This and (23) imply that the curves $\partial^{m} t^{1} / \partial r^{m}(r, s)=$ constant in $W_{2}$ are decreasing. Then there is a unique zero curve $C_{r^{m}}$ of $\partial^{m} t^{1} / \partial r^{m}$ through $R_{0}$, which is decreasing in $W_{2}$. Let $r=\phi(s)$ describe $C_{r^{m}}$. Expanding $\partial^{m} t^{1} / \partial r^{m}(r, a)$ into power series about $r_{0}$ gives $\partial^{m} t^{1} / \partial r^{m}(r, a)=$ $\delta\left(r-r_{0}\right)+o\left(\left|r-r_{0}\right|\right)$. By $(22) \quad \partial^{m} t^{1} / \partial r^{m}(r, s)>0(<0)$ for $(r, s) \in W_{2}$ such that $r<\phi(s)(r>\phi(s))$. It follows from (23) that there is a unique zero curve $C_{r^{m-1}}$ of $\partial^{m-1} t^{1} / \partial r^{m-1}$ in $W_{2}$, which is of the first kind through $R_{0}$, with $R_{0}$ as a bending maximum point. Let $s=\tilde{\phi}(r)$ describe $C_{r^{m-1}}$. We also find that $\partial^{m-1} t^{1} / \partial r^{m-1}(r, s)<0(>0)$ for $(r, s) \in W_{2}$ such that $s>\tilde{\phi}(r)(s<\tilde{\phi}(r))$. Applying similar argument repeatedly we can derive that $C_{r}$ is of the first kind through $R_{0}$ with the other properties listed in (ii).

By (i) and (21) we have that (iii) holds.

For Case 3, given $R_{0} \in\left(A_{s} \cap C_{0} \cap \Omega_{2}\right)-A_{r}$, by (20) and applying the result of Case 1 we find that there is a unique zero curve $C_{s}$ of $t_{s}$ as shown in Fig. 3, which is of the first kind through $R_{0}$, with $R_{0}$ as a bending maximum point and that $R_{0}$ is an isolated point of $\partial N \cap C_{0}$.

For Case 4, given $R_{0} \in\left(A_{s} \cap C_{0} \cap\{r=-a\}\right)-\left(A_{r} \cup V\right)$, by (20) and applying Lemma 12 (i), (iii) we find that there is a deleted neighborhood of $R_{0}$ in $\Omega_{2}$ in which $t_{s}>0$ and $t_{r}<0$ and that $R_{0}$ is an isolated point of $\partial N \cap C_{0}$. By (18) $t_{2 s}^{1}(-a, s)=t_{s}(-a, s)(-a \leqq s \leqq a)$ while by $(19) t_{2 s}^{1}(r, s)=-t_{r}^{1}(-s,-r)$. Applying Lemma 12 (ii) we find that there is a unique zero curve $C_{s}$ of $t_{2 s}^{1}$, which is of the first kind through $R_{0}$, with $R_{0}$ as a bending minimum point. Sup- 
pose $r=r(s)$ describes $C_{s}$. We also have that $t_{2 s}^{1}(r, s)>0(<0)$ for $r<r(s)(r>r(s))$ and $\left|s-s_{0}\right|$ small.

For Case 5 we prove

Lemma 13. Given $R_{0}=\left(r_{0}, s_{0}\right) \in A_{r} \cap A_{s} \cap C_{0} \cap \check{\Omega}_{2}$, the following hold.

(i) There is a convex neighborhood $W$ of $R_{0}$ in $\AA_{2}$ such that $t_{s}>t_{r}$ in $W-\left\{R_{0}\right\}$.

(ii) There is a unique zero curve $C_{r}\left(C_{s}\right)$ of $t_{r}\left(t_{s}\right)$, which is of the first kind through $R_{0}$, with $R_{0}$ as a bending minimum (maximum) point.

(iii) Suppose $s=s(r)(r=r(s))$ describes $C_{r}\left(C_{s}\right)$. The function $s=s(r) \quad(r=r(s))$ is smooth everywhere except perhaps at $r=r_{0}$ $\left(s=s_{0}\right)$. For $s<s(r)(s>s(r)),\left|r-r_{0}\right|$ and $\left|s-s_{0}\right|$ small, $t_{r}(r, s)<0$ $(>0)$. For $r>r(s)(r<r,(s)),\left|r-r_{0}\right|$ and $\left|s-s_{0}\right| s m a l l, t_{s}(r, s)>0$ $(<0)$.

(iv) $C_{s}$ lies to the left of $C_{r}$ as shown in Fig. 4.

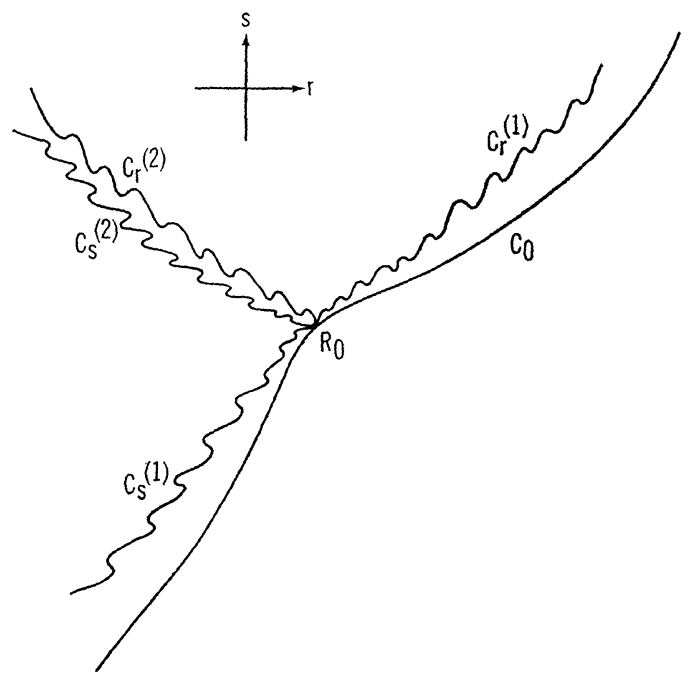

Figure 4

(v) The point $R_{0}$ is an isolated point of $\partial N \cap C_{0}$.

Proof. By Lemma 10 (i), (iii) and Lemma 9 (i)

(24) $t_{r}\left(r, s_{0}\right)<0$ for $r_{0}<r \leqq s_{0}$ and $t_{s}\left(r_{0}, s\right)>0$ for $r_{0} \leqq s<s_{0}$.

Since $t(r, s)$ is analytic, there are positive integers $m$ and $n$ such that

$$
\begin{aligned}
& \partial^{i} t_{r} / \partial r^{i}\left(R_{0}\right)=0(0 \leqq i \leqq m-1), \alpha_{0}=\partial^{m} t_{r} / \partial r^{m}\left(R_{0}\right) \neq 0 \\
& \partial^{j} t_{s} / \partial s^{j}\left(R_{0}\right)=0(0 \leqq j \leqq n-1), \beta_{0}=\partial^{n} t_{s} / \partial s^{n}\left(R_{0}\right) \neq 0 .
\end{aligned}
$$


Using (4) and (25) we can show by induction that

$$
\partial^{i+j} t / \partial r^{i} \partial s^{j}\left(R_{0}\right)=0(1 \leqq i \leqq m ; 1 \leqq j \leqq n) .
$$

Expanding $t(r, s)$ into power series about $R_{0}$ and applying (26) give

$$
\begin{gathered}
t(r, s)=T_{0}+\left(\alpha_{0} /(m+1) !\right)\left(r-r_{0}\right)^{m+1}+\left(\beta_{0} /(n+1) !\right)\left(s-s_{0}\right)^{n+1} \\
+o\left(\left|r-r_{0}\right|^{m+1}\right)+o\left(\left|s-s_{0}\right|^{n+1}\right) .
\end{gathered}
$$

Differentiating (4) and using (25) and (26) we obtain

$$
\begin{aligned}
& \alpha_{1}=\partial^{m+1} t_{s} / \partial r^{m+1}\left(R_{0}\right)=\rho\left(r_{0}-s_{0}\right) \alpha_{0} \neq 0, \\
& \beta_{1}=\partial^{n+1} t_{r} / \partial s^{n+1}\left(R_{0}\right)=-\rho\left(r_{0}-s_{0}\right) \beta_{0} \neq 0 .
\end{aligned}
$$

Expanding $t_{r}$ and $t_{s}$ into power series about $R_{0}$ and applying (25), (26), and (28) give

$$
\begin{aligned}
t_{r}(r, s)=( & \left.\alpha_{0} / m !\right)\left(r-r_{0}\right)^{m}+\left(\beta_{1} /(n+1) !\right)\left(s-s_{0}\right)^{n+1}+o\left(\left|r-r_{0}\right|^{m}\right) \\
& +o\left(\left|s-s_{0}\right|^{n+1}\right), \\
t_{s}(r, s)= & \left(\beta_{0} / n !\right)\left(s-s_{0}\right)^{n}+\left(\alpha_{1} /(m+1) !\right)\left(r-r_{0}\right)^{m+1} \\
& +o\left(\left|s-s_{0}\right|^{n}\right)+o\left(\left|r-r_{0}\right|^{m+1}\right) .
\end{aligned}
$$

Recall that $t\left(r_{0}(s), s\right) \equiv T_{0}$, where $r=r_{0}(s)$ describes $C_{0}$. By the continuity of $t_{r}$ and by Lemma 11, given any $\eta>0$, there is a point $\left(r_{i}, s_{i}\right)$ lying to the left of $C_{0}, 0<(-1)^{i}\left(r_{0}-r_{i}\right)<\eta$ and $0<(-1)^{i}$ $\left(s_{0}-s_{i}\right)<\eta$, such that the line segments $\left\{\left(r, s_{i}\right): r_{i} \leqq r \leqq r_{0}\left(s_{i}\right)\right\}$ lie in $N(i=1,2)$. Then by Lemma $9(\mathrm{i})$

$$
t\left(r_{i}, s_{i}\right)>T_{0}(i=1,2) .
$$

By (24), (29), (27), and (30) we can show that $m$ and $n$ are even numbers. It follows from (24) and (29) that

$$
\alpha_{0}<0 \text { and } \beta_{0}>0 \text {. }
$$

By (29) and (31) we can derive (i).

Differentiating (4) and using (7), (25), (26), (31) we obtain

$$
\begin{gathered}
(-1)^{j+1} \alpha_{j}=(-1)^{j+1} \partial^{j+m+1} t / \partial r^{m+1} \partial s^{j}\left(R_{0}\right)>0(1 \leqq j \leqq n), \\
\beta_{i}=\partial^{i+n+1} t / \partial r^{i} \partial s^{n+1}\left(R_{0}\right)>0(1 \leqq i \leqq m)
\end{gathered}
$$

Expanding $\partial^{m} t_{s} / \partial r^{m}$ into power series about $R_{0}$ gives

$$
\begin{array}{r}
\partial^{m} t_{s} / \partial r^{m}(r, s)=\alpha_{1}\left(r-r_{0}\right)+\left(\beta_{m} / n !\right)\left(s-s_{0}\right)^{n} \\
+o\left(\left|r-r_{0}\right|\right)+o\left(\left|s-s_{0}\right|^{n}\right) .
\end{array}
$$

It follows from (32) that $\partial^{m} t_{s} / \partial r^{m}(r, s)>0$ for small positive $r-r_{0}$ and $\left|s-s_{0}\right|$. This and (31) imply that for small nonnegative $s-s_{0}$ there is a unique zero curve $C_{r^{m}}$ of $\partial^{m} t / \partial r^{m}$ through $R_{0}$, which is 
increasing. Let $r=\phi(s), s \geqq s_{0}$, describe $C_{r^{m}}$. By (31)

$$
\partial^{m} t / \partial r^{m}(r, s)>0 \text { for } r<\phi(s),\left|r-r_{0}\right| \text { and } s-s_{0} \text { small, }
$$

and

(33) $\partial^{m} t / \partial r^{m}(r, s)<0 \quad$ for $\quad r>\phi(s), r-r_{0} \quad$ and $\quad s-s_{0} \quad$ small .

Expanding $\partial^{m-1} t_{s} / \partial r^{m-1}$ into power series about $R_{0}$ and applying (32) give that $\partial^{m-1} t_{s} / \partial r^{m-1}>0$ in a deleted neighborhood of $R_{0}$. This and (33) imply that for small nonnegative $s-s_{0}$ there is a unique zero curve $C_{r^{m-1}}$ of $\partial^{m-1} t / \partial r^{m-1}$, which is of the first kind through $R_{0}$, with $R_{0}$ as a bending minimum point. Let $s=\tilde{\phi}(r)$ describe $C_{r^{m-1}}$. By (33) we have that $\partial^{m-1} t / \partial r^{m-1}(r, s)<0(>0)$ for $s_{0} \leqq s<\tilde{\phi}(r)(s>\tilde{\phi}(r)),\left|r-r_{0}\right|$ and $s-s_{0}$ small. Applying similar argument repeatedly we can derive that there is a unique zero curve $C_{r}$ of $t_{r}$, which is of the first kind through $R_{0}$, described by a function $s=s(r)$ with $R_{0}$ as a bending minimum point and that $t_{r}(r, s)<0(>0)$ for $s_{0} \leqq s<s\left(r^{r}\right)\left(s>s\left(r^{*}\right)\right),\left|r-r_{0}\right|$ and $s-s_{0}$ small. By (29), (31), and (32) we also have that $t_{r}(r, s)<0$ for $s<s_{0}$, $\left|r-r_{0}\right|$ and $s_{0}-s$ small. This derives the first parts of (ii) and (iii). Similarly we can drive the remaining parts of (ii) and (iii).

The part (iv) follows from (i), (ii), and (iii), and the part (v) follows from (ii) and (21).

\section{Lemma 14. The set $\partial N \cap C_{0}$ is finite.}

Proof. According to the discussions of Cases 1, 3, and 4 and Lemma 12 (iii) for Case 2 and Lemma 13 (v) for Case 5, we find that both the sets $A_{r} \cap C_{0}$ and $A_{s} \cap C_{0}$ contain only isolated points of $\partial N \cap C_{0}$. It follows from (21) that $\partial N \cap C_{0}$ contains only isolated points. This and the Bolzano-Weierstrass theorem imply that $\partial N \cap C_{0}$ is finite.

We now drop the condition (7) $(i>0)$ and assume only (7) $(i=0)$. Lemmas $7,8,9,10$, and 11 still hold. The results of Cases 1 and 3 also hold.

Presently for Case 2 we are unable to exclude the possibility that the zero curve $C_{r}$ of $t_{r}^{1}$ in Lemma (12) (ii) contains countably infinitely many bending maximum points in any neighborhood of $R_{0}$. Likewise for case 4 the zero curve $C_{s}$ of $t_{2 s}^{1}$ may contain countably infinitely many bending minimum points in any neighborhood of $R_{0}$.

Recall that $r=r_{0}(s), s^{*} \leqq s \leqq a$, describes $C_{0}$. By Lemma 11 any subset of the set $\left\{s: s^{*} \leqq s \leqq a ;\left(r_{0}(s), s\right) \in \partial N \cap C_{0}\right\}$ is not a perfect set. For Case 5 there may be three cases. Case 5a. The point $R_{0}$ is an isolated point of $\partial N \cap C_{0}$. Case 5b. There is a (infinite) sequence of isolated points $\left\{R_{k}\right\}$ of $\partial N \cap C_{0}$ convergent to $R_{0}$. More- 
over, the set $\left\{R_{k}\right\}$ is the only subset of $\partial N \cap C_{0}$ with the above property in a neighborhood of $R_{0}$ and $\left\{R_{k}\right\}$ contains no subsequence convergent to a point other than $R_{0}$. Case $5 \mathrm{c}$. There is a sequence of points $\left\{R_{k}\right\}$ of $\partial N \cap C_{0}$ convergent to $R_{0}$ such that for each $k$ the point $R_{k}$ is of Case $5 \mathrm{~b}$. Suppose $\left\{R_{k_{n}}\right\}$ is the sequence of isolated points of $\partial N \cap C_{0}$ convergent to $R_{k}$.

For Case 5a, Lemma 13(i), (iii), (iv), (v) still hold. The proof of Lemma 13 (i) is exactly the same as before. Lemma 13(ii) is modified to:

There is a unique zero curve $C_{r}\left(C_{s}\right)$ of $t_{r}\left(t_{s}\right)$ through $R_{0}$ which lies above $\left\{s=s_{0}\right\}$ (to the left of $\left\{r=r_{0}\right\}$ ).

To verify this statement and Lemma 13(iii), (iv) we use the results obtained in proving Lemma 13(i). By (28), (7) $(i=0)$, and (31)

$$
\alpha_{1}>0 \text { and } \beta_{1}>0 \text {. }
$$

By (29), (31), and (34) we can choose the convex neighborhood $W$ of $R_{0}$ in Lemma 13(i) sufficiently small such that

$$
\begin{aligned}
& t_{r}(r, s)<0 \text { for }(r, s) \in\left\{(r, s) \in W: s \leqq s_{0}\right\}-\left\{R_{0}\right\}, \\
& t_{r}\left(r_{0}, s\right)>0 \text { for }\left(r_{0}, s\right) \in\left\{(r, s) \in W: s>s_{0}\right\}, \\
& t_{s}(r, s)>0 \text { for }(r, s) \in\left\{(r, s) \in W: r \geqq r_{0}\right\}-\left\{R_{0}\right\}, \\
& t_{s}\left(r, s_{0}\right)<0 \text { for }\left(r, s_{0}\right) \in\left\{(r, s) \in W: r<r_{0}\right\} .
\end{aligned}
$$

Now $R_{0}$ is assumed to be an isolated point of $\partial N \cap C_{0}$. By Lemma 10(iii) there exists an arc $\gamma$ on $C_{0}$ containing $R_{0}$ such that $\gamma-\left\{R_{0}\right\} \subset N$. We choose $W$ such that $W \cap C_{0} \subset \gamma$. By Lemma 9(i) $t_{r}<0$ and $t_{s}>0$ along $\left(W \cap C_{0}\right)-\left\{R_{0}\right\}$. Let

$$
\begin{aligned}
& W_{1}=\left\{(r, s) \in W: r>r_{0} ; s>s_{0} ;(r, s) \text { lies to the left of } C_{0}\right\}, \\
& W_{2}=\left\{(r, s) \in W: r<r_{0}, s>s_{0}\right\}, \\
& W_{3}=\left\{(r, s) \in W: r<r_{0} ; s<s_{0} ;(r, s) \text { lies to the left of } C_{0}\right\} .
\end{aligned}
$$

According to (35) $t_{r}$ vanishes somewhere in $W_{1}$ and in $W_{2} ; t_{s}$ vanishes somewhere in $W_{2}$ and in $W_{3}$. By (4), (7) $(i=0)$, and Lemma 13(i),

$$
t_{r s}>0 \text { in } W-\left\{R_{0}\right\} .
$$

It follows from the implicit function theorem that there is a unique $C_{r}=C_{r}^{(1)} \cup C_{r}^{(2)}$, which is described by a function $s=s(r)$ smooth everywhere except perhaps at $r=r_{0}$, through $R_{0}$ such that $C_{r}^{(1)}-$ $\left\{R_{0}\right\} \subset W_{1}$ and $C_{r}^{(2)}-\left\{R_{0}\right\} \subset W_{2}$. There is also a unique $C_{s}=C_{s}^{(1)} \cup C_{s}^{(2)}$, which is described by a function $r=r(s)$ smooth everywhere except perhaps at $s=s_{0}$, through $R_{0}$ such that $C_{s}^{(1)}-\left\{R_{0}\right\} \subset W_{3}$ and $C_{s}^{(2)}-$ $\left\{R_{0}\right\} \subset W_{2}$. This derives the above modified version of Lemma 13 (ii) and the first part of Lemma 13(iii). From (36) the second part of 
Lemma 13 (iii) follows. This and Lemma 13(i) imply Lemma 13(iv). There are two subcases of Case 5a. (i) Both $C_{r}$ and $C_{s}$ are of the first kind through $R_{0}$. (ii) $C_{r}$ (or $C_{s}$ ) contains countably infinitely many bending minimum (or maximum) points in any neighbourhood of $R_{0}$.

For Case $5 \mathrm{~b}$ (Case 5c), for each $k$ (for each $k$ and for each $n$ ) the point $R_{k}\left(R_{k_{n}}\right)$ is of one of the following three cases: (i) $R_{k}\left(R_{k_{n}}\right) \in$ $A_{r}-A_{s}$. This is Case 1. (ii) $R_{k}\left(R_{k_{n}}\right) \in A_{s}-A_{r}$. This is Case 3. (iii) $R_{k}\left(R_{k_{v}}\right) \in A_{r} \cap A_{s}$. This is Case 5a.

4. The characterizations of the breakdown points.

THEOREM 1. Suppose $X(R)$ is a solution of (3) over $\Omega$. Given $a$ point $R_{0}=\left(r_{0}, s_{0}\right)$ in $\Omega,-a<r_{0}<a$ and $-a \leqq s_{0}<a$, assume that there is a zero curve $C_{r}$ of $t_{r}$, which is of the first kind through $R_{0}$, described by a function $s=s(v)$ with $R_{0}$ as a bending minimum point. Assume further that $s(r)$ is smooth everywhere in a neighborhood of $r_{0}$ except perhaps at $r_{0}$. Suppose $W$ is a convex neighborhood of $R_{0}$ in $\Omega$. Let $W_{1}=\{(r, s) \in W: s \geqq s(r)\}$ and $W_{2}=\{(r, s) \in$ $\left.W: s \geqq s_{0}\right\}$. Suppose the following hold.

(i) $t_{r}<0$ in $W_{2}-W_{1}$,

(ii) $t_{s}>0$ in $W_{2}-\left\{R_{0}\right\}$,

(iii) $t_{s}>t_{r}$ (or, by (3), $-x_{s}>x_{r}$ ) in $W_{2}-\left\{R_{0}\right\}$.

Then there is a unique 1-shock originating at $P_{0}=X\left(R_{0}\right)$. Moreover, if $t_{s}\left(R_{0}\right)>0$, then this shock is the only shock curve originating at $P_{0}$.

By a method similar to that used in proving Theorem 4.3 of [2] we can prove Theorem 1 .

Definition 2. Let $\mathfrak{I}_{B}=\left\{\left(x, T_{0}\right): 0 \leqq x \leqq 1\right\}, T_{0}>0$, be the line segment at which the solution $U$ breaks down. Suppose $\gamma$ is a segment of $\mathfrak{l}_{B}$. Then $\gamma$ is strongly regular (s.r.) if it contains no b.d.p.; $\gamma$ is weakly regular (w.r.) if it contains only finitely many b.d.p.'s, each of which is of one of the kinds I and II as stated in $\S 1$; $\gamma$ is regular (r.) if it is either s.r. or w.r.

THEOREM 2. Under (5), (6), (7), and (8) the segment $\mathfrak{l}_{B} \cap D_{2}^{0}$ is $r$.

Proof. It suffices to assume that the solution of $U$ breaks down in $D_{2}^{0}$ and to prove that $\mathfrak{l}_{B} \cap D_{2}^{0}$ is w.r.

From Lemma 14, $\mathfrak{l}_{B} \cap D_{2}^{\circ}$ contains only finitely many b.d.p.'s. To show that each of them is of one of the kinds I and II we con- 
sider the five possible cases in $\S 3$. Let $P_{0}=X_{2}^{0}\left(R_{0}\right)$.

For Case 1 by Theorem 1 there is a unique shock curve originating at $P_{0}$, which is an 1-shock. Then $P_{0}$ is of the kind I. For Case 3 by (20) and using the result of Case 1 we find that there is a unique shock curve originating at $P_{0}$, which is a 2-shock. Thus $P_{0}$ is of the kind $\mathrm{I}$.

For Case 2, by Lemma 12 (ii) and Lemma 6 (iii) and applying the same methods as those used in proving Theorems 3.1 and 4.3 of [2], we can prove that there is a unique shock curve originating at $P_{0}$, which is an 1-shock. Then $P_{0}$ is of the kind I.

For Case 4, by (19) and applying the above arguments for Case 2 we find that there is a unique shock curve originating at $P_{0}$, which is a 2-shock. Thus $P_{0}$ is of the kind $\mathrm{I}$.

For Case 5, by Lemma 13(ii), (iii), (i) the conditions (i) and (iii) of Theorem 1 hold. We can weaken the condition (ii) of Theorem 1 to: $t_{s}>0$ in $\left\{(r, s) \in W_{2}: r \geqq r_{0}\right.$, or $r>\theta(s)$ for $\left.r<r_{0}\right\}-\left\{R_{0}\right\}$, where $\theta(s)$ is a decreasing function describing a $C_{s}$ in $W_{2}$ through $R_{0}$, and derive the first conclusion of Theorem 1. For Case 5 by Lemma 13 (ii), (iii), (iv) this modified condition holds. Thus there is a unique 1-shock originating at $P_{0}$. By Lemma 13 (ii), (iii) and (20), and applying the above modified version of Theorem 1 we can also prove that there is a unique 2 -shock originating at $P_{0}$. Thus $P_{0}$ is of the kind II.

TheOREM 3. Under (5), (6), (7) (i=0), and (8) the segment $\mathfrak{l}_{B} \cap D_{2}^{0}$ is either r. or with countably many b.d.p.'s, each of which is of one of the four kinds as stated in $\S 1$.

Proof. We assume that the solution $U$ breaks down in $D_{2}^{\circ}$ and consider the five possible cases in $\S 3$. Let $P_{0}=X_{2}^{\circ}\left(R_{0}\right)$.

As we observed at the end of $\S 3$ the results of Cases 1 and 3 still hold. For each of these two cases, from the proof of Theorem 2 we can verify that $P_{0}$ is of the kind $I$.

For Case 2 since the zero curve $C_{r}$ of $t_{r}^{1}$ either is of the first kind through $R_{0}$ or contains countably infinitely many bending maximum points in any neighborhood of $R_{0}$, from the proof of Theorem 2 we can verify that $P_{0}$ is of one of the kinds I and III. Similarly for Case 4, $P_{0}$ is of one of the kinds I and III.

For Case $5 \mathrm{a}(\mathrm{i})$, by the proof of Theorem 2, $P_{0}$ is of the kind II. For Case 5a(ii), by the proof of Theorem 2 we can show that $P_{0}$ is of the kind III. For Case 5b (5c) from the above discussions of Cases 1,3 , and $5 \mathrm{a}$ we can see that $P_{0}$ is of one of the kinds III and IV (of the kind IV). 
Definition 3. For $k \geqq 0$ and $i=1,2$, the initial signs (i.s.) of $t_{i r}^{2 k}$ and $t_{i s}^{2 k}\left(t_{r}^{2 k+1}\right.$ and $\left.t_{s}^{2 k+1}\right)$ are their own signs if $D_{i}^{2 k}\left(D^{2 k+1}\right)$ lies strictly below $\mathfrak{l}_{B}$, or their original signs before the occurence of the changes of signs if $\mathfrak{l}_{B}$ intersects $D_{i}^{2 k}\left(D^{2 k+1}\right)$; the initial signs distribution (i.s.d.) of a region $D_{i}^{2 k}\left(D^{2 k+1}\right)$ is $(+,-)$ if the i.s.'s of $t_{i r}^{2 k}$ and $t_{i s}^{2 k}$ $\left(t_{r}^{2 k+1}\right.$ and $\left.t_{s}^{2 k+1}\right)$ are + and - respectively. We define the i.s.d.'s $(-,+)$, $(-,-)$, and $(+,+)$ similarly.

\section{By Lemma 6(ii), (iii) we have}

LeMma 15 . For $k \geqq 0$ if the region $D_{\mathrm{I}}^{4 k}\left(D_{2}^{4 k}, D^{4 k+1}, D_{1}^{4 k+2}, D_{2}^{4 k+2}\right.$, $D^{4 k+3}$ respectively) either lies strictly below $\mathfrak{l}_{B}$ or intersects $\mathfrak{l}_{B}$, then its i.s.d. is $(+,-)((-,+),(-,-),(-,+),(+,-),(+,+)$ respectively).

THEOREM 4. Under (5), (6), (7), and (8) the segment $\mathfrak{l}_{B}$ is w.r.

Proof. We consider first the following four cases: Case 1. $\mathfrak{l}_{B} \subset D_{1}^{0} \cup D^{1} \cup D_{2}^{0}$, Case 2. $\mathfrak{l}_{B} \subset D_{1}^{2} \cup D^{1} \cup D_{2}^{2}$, Case 3. $\mathfrak{l}_{B} \subset D_{1}^{2} \cup D^{3} \cup D_{2}^{2}$, Case 4. $\mathfrak{l}_{B} \subset D_{1}^{4} \cup D^{3} \cup D_{2}^{4}$.

Case 1. We have observed in $\S 3$ that $\mathfrak{l}_{B} \cap D_{1}^{0}$ is s.r.. By Theorem $2, \mathfrak{l}_{B} \cap D_{2}^{0}$ is $\mathrm{r}$.

By Lemma 15 the i.s.d. of $D^{1}$ is $(-,-)$. As we construct the set $N$ in $\S 3$ we construct the set $N^{1}$ which is the largest subset of $\Omega$ satisfying (i) $N^{1}$ is open and connected, (ii) $N^{1}$ contains the vertex $(a, a)$, (iii) $t_{r}^{1}<0$ and $t_{s}^{1}<0$ in $N^{1}$. Also as we construct the curve $C_{0}$ in $\S 3$ we construct the curve $C_{0}^{1}$ in $\Omega$ such that $X^{1}\left(C_{0}^{1}\right)=$ $\left(\mathfrak{l}_{B} \cap D^{1}\right)-\left(D_{1}^{0} \cup D_{2}^{0}\right)$. The curve $C_{0}^{1}$ satisfies: (i) $C_{0}^{1}$ is decreasing. (ii) The interior of the region to the right of $C_{0}^{1}$ in $\Omega$ is contained in $N^{1}$. Suppose the solution $U$ breaks down at $\left(\mathfrak{l}_{B} \cap D^{1}\right)-\left(D_{1}^{0} \cup D_{2}^{0}\right)$. Then $N^{1} \varsubsetneqq \Omega$; if $R \in \partial N^{1}, t_{r}^{1}(R)=0$ or $t_{s}^{1}(R)=0 ; \partial N^{1} \cap C_{0}^{1} \neq \varnothing$. Let $A_{r}^{1}=\{R \in$ $\left.\Omega ; t_{r}^{1}(R)=0\right\}$. The condition $(7)(i=0)$ implies that $q^{*}(r-s)>0$ for $(r, s) \in \Omega$. By (11) we can derive that $t_{s}^{1}<0$ along $C_{0}$ and so that $\partial N^{1} \cap C_{0}^{1}=A_{r}^{1} \cap C_{0}^{1}$. Given any point $R_{0} \in A_{r}^{1} \cap C_{0}^{1}$, by (4) and the implicit function theorem we derive the same conclusion of Lemma 12(ii). Applying the same methods as those used in proving Theorems 3.1 and 4.3 of [2] we can prove that at $X^{1}\left(R_{0}\right)$ there originates a unique shock curve, which is an 1-shock. Thus the b.d.p.'s on $\left(\mathfrak{I}_{B} \cap D^{1}\right)-\left(D_{1}^{0} \cup D_{2}^{0}\right)$ are of the kind I. By the method used in proving Lemma 14 we can prove that $\partial N^{1} \cap C_{0}^{1}$ is finite. That is, the segment $\left(\mathfrak{l}_{B} \cap D^{1}\right)-\left(D_{1}^{0} \cup D_{2}^{0}\right)$ contains only finitely many b.d.p.'s. We recall that we assumed that $U$ breaks down at $\left(\mathfrak{l}_{B} \cap D^{1}\right)-\left(D_{1}^{0} \cup D_{2}^{0}\right)$. It may not break down there. Thus $\left(\mathfrak{l}_{B} \cap D^{1}\right)-$ 
$\left(D_{1}^{0} \cup D_{2}^{0}\right)$ is $\mathbf{r}$. It follows that $\mathfrak{l}_{B}$ is w.r.

Case 2. By the same argument used in Case 1 we can show that $\left(\mathfrak{l}_{B} \cap D^{1}\right)-\left(D_{1}^{2} \cup D_{2}^{2}\right)$ is $\mathrm{r}$.

By Lemma 15 the i.s.d. of $D_{2}^{2}$ is $(+,-)$. We construct the set $N_{2}^{2}$ which is the largest subset of $\Omega$ satisfying (i) $N_{2}^{2}$ is open and connected, (ii) $N_{2}^{2}$ contains the vertex $(-a, a)$, (iii) $t_{2 r}^{2}>0$ and $t_{2 s}^{2}<0$ in $N_{2}^{2}$. We also construct the curve $C_{2,0}^{2}$ in $\Omega$ such that $\{(x, t) \in$ $\left.X_{2}^{2}\left(C_{2,0}^{2}\right): x \leqq 1\right\}=\mathfrak{l}_{B} \cap D_{2}^{2}$. The curve $C_{2,0}^{2}$ satisfies: (i) $C_{2,0}^{2}$ is increasing. (ii) The interior of the region to the left of $C_{2,0}^{2}$ in $\Omega$ is contained in $N_{2}^{2}$. By (19) the curve $C_{2,0}^{2}$ is symmetric with respect to the diagonal $r=-s$. Let $R_{1}=\left(r_{0}, a\right)$ and $R_{2}=\left(-a,-r_{0}\right),-a<r_{0}<a$, be the points of intersection of $C_{2,0}^{2}$ with $\{s=a\}$ and $\{r=-a\}$. Now $R_{2}$ is one of the end points of the curve $C_{0}^{1}$ constructed in Case 1 over which $t_{s}^{1}<0$. It follows from (18) that $t_{2 s}^{2}\left(R_{2}\right)=t_{s}^{1}\left(R_{2}\right)<0$. By (19) $t_{2 r}^{2}\left(R_{1}\right)=-t_{2 s}^{2}\left(R_{2}\right)>0$. Combining this with the fact that the interior of the region to the left of $C_{2,0}^{2}$ in $\Omega$ is contained in $N_{2}^{2}$ we find that $t_{2 r}^{2}(r, a)>0$ for $-a \leqq r \leqq r_{0}$ and $t_{2 s}^{2}(-a, s)<0$ for $-r_{0} \leqq s \leqq a$. Let $\hat{\Omega}$ be the square with vertices $(-a, a), R_{1},\left(r_{0},-r_{0}\right)$ and $R_{2}$. Integrating (10) and (11) with the sides $\left\{(r, a):-a \leqq r \leqq r_{0}\right\}$ and $\left\{(-a, s):-r_{0} \leqq s \leqq a\right\}$ as initial curves and applying successive approximations we can prove that $t_{2 r}^{2}>0$ and $t_{2 s}^{2}<0$ in $\hat{\Omega}$. Now the curve $C_{2,0}^{2}$ is contained in $\hat{\Omega}$. It follows from Lemmas 2 and 3 that $\mathfrak{l}_{B} \cap D_{2}^{2}$ is s.r.

By Lemma 15 the i.s.d. of $D_{1}^{2}$ is $(-,+)$ which is the same as that of $D_{2}^{1}$. By a method similar to that used in proving Theorem 2 we can prove that $\mathfrak{l}_{B} \cap D_{1}^{2}$ is r. It follows that $\mathfrak{l}_{B}$ is w.r.

Case 3. As in Case 2, $\mathfrak{l}_{B} \cap D_{2}^{2}$ is s.r. and $\mathfrak{l}_{B} \cap D_{1}^{2}$ is r.

By Lemma 15 the i.s.d. of $D^{3}$ is $(+,+)$. We construct the set $N^{3}$ which is the largest subset of $\Omega$ satisfying (i) $N^{3}$ is open and connected, (ii) $N^{3}$ contains the vertex $(-a,-a)$, (iii) $t_{r}^{3}>0$ and $t_{s}^{3}>0$ in $N^{3}$. We also construct the curve $C_{0}^{3}$ in $\Omega$ such that $X^{3}\left(C_{0}^{3}\right)=$ $\left(\mathfrak{l}_{B} \cap D^{3}\right)-\left(D_{1}^{2} \cup D_{2}^{2}\right)$. The curve $C_{0}^{3}$ satisfies (i) $C_{0}^{3}$ is decreasing. (ii) The interior of the region to the left of $C_{0}^{3}$ in $\Omega$ is contained in $N^{3}$. Suppose $U$ breaks down at $\left(\mathfrak{l}_{B} \cap D^{3}\right)-\left(D_{1}^{2} \cup D_{2}^{2}\right)$. Then $N^{3} \varsubsetneqq \Omega$; if $R \in \partial N^{3}, t_{r}^{3}(R)=0 \quad$ or $\quad t_{s}^{3}(R)=0 ; \partial N^{3} \cap C_{0}^{3} \neq \varnothing$. Let $A_{s}^{3}=\{R \in \Omega$ : $\left.t_{s}^{3}(R)=0\right\}$. By (10) we can derive that $t_{r}^{3}>0$ along $C_{0}^{3}$ so that $\partial N^{3} \cap$ $C_{0}^{3}=A_{s}^{3} \cap C_{0}^{3}$. Given any point $R_{0}=\left(r_{0}, s_{0}\right) \in A_{s}^{3} \cap C_{0}^{3}$, by (4) and the implicit function theorem we can derive that there is a unique zero curve $C_{s}$ of $t_{s}^{3}$, which is of the first kind through $R_{0}$, with $R_{0}$ as a bending minimum point. Suppose $r=r(s)$ describes $C_{s}$. We also have that $t_{s}^{3}(r, s)>0(<0)$ for $r<r(s)(r>r(s))$ and $\left|s-s_{0}\right|$ small. 
Applying methods similar to those used in proving Theorems 3.1 and 4.3 of [2] we can prove that at $X^{3}\left(R_{0}\right)$ there originates a unique shock curve, which is a 2-shock. Thus the b.d.p.'s on $\left(\mathfrak{l}_{B} \cap D^{3}\right)-\left(D_{1}^{2} \cup D_{2}^{2}\right)$ are of the kind I. By the method used in proving Lemma 14 we can prove that $\partial N^{3} \cap C_{0}^{3}$ is finite. Now $U$ may not break down at $\left(\mathfrak{l}_{B} \cap D^{3}\right)-\left(D_{1}^{2} \cup D_{2}^{2}\right)$. Thus this segment is $\mathbf{r}$. It follows that $\mathfrak{l}_{B}$ is w.r.

Case 4. As in Case 3, $\left(\mathfrak{l}_{B} \cap D^{3}\right)-\left(D_{1}^{4} \cup D_{2}^{4}\right)$ is $\mathrm{r}$.

By Lemma 15 the i.s.d.'s of $D_{1}^{4}$ and $D_{2}^{4}$ are $(+,-)$ and $(-,+)$ respectively. By a method similar to that used in showing that $\mathfrak{l}_{B} \cap D_{2}^{2}$ is s.r. in Case 2 we can show that $\mathfrak{l}_{B} \cap D_{1}^{4}$ is s.r. By the same method as that used in showing Theorem 2 we can show that $\mathfrak{l}_{B} \cap D_{2}^{4}$ is r. Thus $\mathfrak{l}_{B}$ is w.r.

Suppose in general $\mathfrak{l}_{B}$ is contained in $D_{1}^{2 n} \cup D^{2 n+1} \cup D_{2}^{2 n}\left(D_{1}^{2 n+2} \cup\right.$ $\left.D^{2 n+1} \cup D_{2}^{2 n+2}\right), n \geqq 2$. By Lemma 15 the i.s.d.'s of $D_{i}^{4 k+j}$ and $D^{4 k+1}$ are the same as those of $D_{i}^{j}$ and $D^{r}$ respectively, $i=1,2 ; k \geqq 1$; $j=0,2 ; \mathfrak{l}=1,3 ; 4 k+j \leqq 2 n(4 k+j \leqq 2 n+2) ; 4 k+\mathfrak{l} \leqq 2 n+1$. We can apply the arguments used in the above four cases to derive that $\mathfrak{l}_{B}$ is w.r.

THEOREM 5. Under (5), (6), (7) $(i=0)$, and (8) the segment $\mathfrak{l}_{B}$ is either w.r. or with countably many b.d.p.'s, each of which is of one of the four kinds as stated in $\S 1$.

Applying the arguments used in the proofs of Theorems 3 and 4 we can prove Theorem 5 .

REMARK 1. The methods used in this paper are applicable to the initial value problem of (1) with initial conditions $u(x, 0)=g(x)$, $v(x, 0)=f(x),-\infty<x<\infty$, where $f(g)$ is an odd (even) periodic analytic function with period $2 ; f^{\prime}(x)+Q(g(x)) g^{\prime}(x)$ and $f^{\prime}(x)-$ $Q(g(x)) g^{\prime}(x)$ vanish on only two finite sets contained in $[0,1]$. The existence of a local solution and certain recursion formulas similar to Lemma 5 can be derived by the methods of MacCamy and Mizel [10] (see Remark 3 of [1] about the existence of a local solution). We observe from the proof of Theorem 4 that the i.s.d. plays the main role in characterizing the b.d.p.'s. There are four possible distributions $(+,-),(-,+),(-,-)$, and $(+,+)$, which all appeared in the proof of Theorem 4. For the general problem using the recursion formulas similar to Lemma 5 we can derive a lemma similar to Lemma 6, which determines the i.s.d.'s similar to those in Lemma 15. Then the methods used in $\S \S 3$ and 4 can be applied to derive 
the conclusion of Theorem 4 under (7) and that of Theorem 5 under (7) $(i=0)$.

REMARK 2. Equations (1) govern the motion of an isentropic gas, where $u$ is the specific volume, $v$ the velocity, and $-\int Q^{2}(u) d u$ the pressure. For a polytropic gas $Q(u)=c^{2} u^{\left(-\gamma^{-1) / 2}\right.}, \gamma>1$, so that the condition (7) holds.

REMARK 3. We may replace the condition (7) by (37) $\partial^{i} \rho / \partial s^{i}(r-s)=(-1)^{i} \partial^{i} \rho / \partial r^{2}(r-s)>0$ for $i \geqq 0$ and $(r, s) \in \Omega$.

By applying the same methods as those used in $\S \S 3$ and 4 we can derive the conclusion of Theorem 4 under the further conditions imposed on the initial functions as in Remark 1. The second order quasilinear wave equation $y_{t t}-\left(1+\varepsilon y_{x}\right)^{\alpha} y_{x x}=0$ (see [12]), where $\alpha$ and $\varepsilon$ are positive constants, is equivalent to (1) if we set $u=y_{x}$, $v=y_{t}$, and $Q(u)=(1+\varepsilon u)^{\alpha / 2}$. For this system the condition (37) holds.

REMARK 4. The conditions of analyticity on $Q$ and $f$ assumed in (5) and (6) were used in the proofs of Lemmas 7 and 11 to exclude the possibility that $C_{r}$ or $C_{s}$ contains horizontal or vertical segments. They were also used in the proofs of Lemmas 12 and 13 to initiate the Taylor's series arguments. Now we weaken these conditions to those of $C^{2}$. Assume that $U$ breaks down in $D_{2}^{0}$. From (21), $\partial N \cap C_{0}=\left(A_{r} \cap C_{0}\right) \cup\left(A_{s} \cap C_{0}\right)$. Suppose $\partial N \cap C_{0}$ contains a horizontal segment. Since $t_{2}^{0}(r, s) \equiv T_{0}$ along $C_{0}, t_{2 r}^{2} \equiv 0$ along this segment. It follows from (3) that the $X_{2}^{0}$ image of this segment is a point. Similarly the $X_{2}^{0}$ image of a vertical segment on $\partial N \cap C_{0}$ is a point. These indicate that the method used in showing the existence of shock curves originating at b.d.p.'s on $\mathfrak{l}_{B} \cap D_{2}^{0}$ may be applied to this problem. Under the further conditions $(7)(i=0)$ and (8) we can derive the conclusion of Theorem 5. The derivation depends heavily on the use of (10) and (11), which replaces that of the Taylor's series arguments. As for the problem considered in Remark 1, weakening the conditions of analyticity on $Q, f$, and $g$ to those of $C^{2}$ and assuming (7) $(i=0)$ we can also derive the conclusion of Theorem 5. For brevity we do not pursue these problems here.

\section{REFERENCES}

1. P. H. Chang, On the breakdown phenomena of solutions of quasilinear wave equations, Michigan Math. J., 23 (1976), 277-287. 
2. P. H. Chang, On the existence of shock curves of quasilinear wave equations, Indiana Univ. Math. J., 26 (1977), 605-622.

3. J. Glimm, Solutions in the large for nonlinear hyperbolic systems of equations, Comm. Pure Appl. Math., 18 (1965), 697-715.

4. J. Glimm and P. D. Lax, Decay of solutions of systems of nonlinear hyperbolic conservation laws, Memoirs of the Amer. Math. Soc., 101, Amer. Math. Soc. Providence, R. I., (1970).

5. A. Jeffrey, The development of singularities of solutions of nonlinear hyperbolic equations of order greater than unity, J. Math. Mech., 15 (1966), 585-598.

6. F. John, Formation of singularities in one-dimensional nonlinear wave propagation, Comm. Pure Appl. Math., 27 (1974), 377-405.

7. Delayed singularity formation in solutions of nonlinear wave equations in higher dimensions, Comm. Pure Appl. Math., 29 (1976), 649-682.

8. P. D. Lax, Hyperbolic systems of conservation laws II, Comm. Pure Appl. Math., 10 (1957), 537-566.

9. ㄴ Development of singularities of solutions of nonlinear hyperbolic partial differential equations, J. Mathematical Phys., 5 (1964), 611-613.

10. R. C. MacCamy and V. J. Mizel, Existence and nonexistence in the large of solutions of quasilinear wave equations, Arch. Rational Mech. Anal., 25 (1967), 299-320.

11. T. Nishida and J. Smoller, Mixed problems for nonlinear conservative laws, J. Differntial Equations, 23 (1977), 244-269.

12. N. J. Zabusky, Exact solution for the vibrations of a nonlinear continuous model string, J. Mathematical Phys., 3 (1962), 1028-1039.

Received November 21, 1977 and in revised form December 29, 1978. This research was supported in part by Natural Sciences and Engineering Research Council of Canada through a grant to Professor D. Naylor and in part by the Faculty of Science of the University of Western Ontario.

The University of Western ONTARo

London, Ontario N6A 5B9

CANADA

AND

IOWA STATE UNIVERSITY

AMEs, IA 50011 



\section{PACIFIC JOURNAL OF MATHEMATICS}

\section{EDITORS}

DONALD BABBITT (Managing Editor)

University of California

Los Angeles, CA 90024

HUgo RossI

University of Utah

Salt Lake City, UT 84112

C. C. Moore and ANDrew OGG

University of California

Berkeley, CA 94720

\section{J. DUGUNDJI}

Department of Mathematics

University of Southern California

Los Angeles, CA 90007

R. FinN and J. MILGRaM

Stanford University

Stanford, CA 94305

\section{ASSOCIATE EDITORS}
E. F. BECKENBACH
B. H. NeumanN
F. WOLF
K. YOSHIDA

\section{SUPPORTING INSTITUTIONS}

UNIVERSITY OF BRITISH COLUMBIA CALIFORNIA INSTITUTE OF TECHNOLOGY

UNIVERSITY OF CALIFORNIA

MONTANA STATE UNIVERSITY

UNIVERSITY OF NEVADA, RENO

NEW MEXICO STATE UNIVERSITY

OREGON STATE UNIVERSITY

UNIVERSITY OF OREGON
UNIVERSITY OF SOUTHERN CALIFORNIA

STANFORD UNIVERSITY

UNIVERSITY OF HAWAII

UNIVERSITY OF TOKYO

UNIVERSITY OF UTAH

WASHINGTON STATE UNIVERSITY

UNIVERSITY OF WASHINGTON

The Supporting Institutions listed above contribute to the cost of publication of this Journal, but they are not owners or publishers and have no responsibility for its content or policies.

Mathematical papers intended for publication in the Pacific Journal of Mathematics should be in typed form or offset-reproduced, (not dittoed), double spaced with large margins. Please do not use built up fractions in the text of the manuscript. However, you may use them in the displayed equations. Underline Greek letters in red, German in green, and script in blue. The first paragraph or two must be capable of being used separately as a synopsis of the entire paper. Please propose a heading for the odd numbered pages of less than 35 characters. Manuscripts, in triplicate, may be sent to any one of the editors. Please classify according to the scheme of Math. Reviews, Index to Vol. 39. Supply name and address of author to whom proofs should be sent. All other communications should be addressed to the managing editor, or Elaine Barth, University of California, Los Angeles, California, 90024.

50 reprints to each author are provided free for each article, only if page charges have been substantially paid. Additional copies may be obtained at cost in multiples of 50 .

The Pacific Journal of Mathematics is issued monthly as of January 1966. Regular subscription rate: $\$ 84.00$ a year (6 Vols., 12 issues). Special rate: $\$ 42.00$ a year to individual members of supporting institutions.

Subscriptions, orders for numbers issued in the last three calendar years, and changes of address should be sent to Pacific Journal of Mathematics, P.O. Box 969, Carmel Valley, CA 93924, U.S.A. Older back numbers obtainable from Kraus Periodicals Co., Route 100, Millwood, NY 10546.

PUBLISHED BY PACIFIC JOURNAL OF MATHEMATICS, A NON-PROFIT CORPORATION

Printed at Kokusai Bunken Insatsusha (International Academic Printing Co., Ltd.). 8-8, 3-chome, Takadanobaba, Shinjuku-ku, Tokyo 160, Japan.

Copyright (C) 1979 by Pacific Journal of Mathematics Manufactured and first issued in Japan 


\section{Pacific Journal of Mathematics}

\section{Vol. 85, No. $2 \quad$ October, 1979}

Charles A. Akemann and Steve Wright, Compact and weakly compact derivations of $C^{*}$-algebras ........................... 253

Dwight Richard Bean, Andrzej Ehrenfeucht and George Frank McNulty, Avoidable patterns in strings of symbols ................... 261

Richard Clark Brown, Notes on generalized boundary value problems in Banach spaces. I. Adjoint and extension theory.................

Kenneth Alexander Brown and John William Lawrence, Injective hulls of group rings .................................... 323

Jacob Burbea, The Schwarzian derivative and the Poincaré metric ....... 345

Stefan Andrus Burr, On the completeness of sequences of perturbed polynomial values ....................................

Peter H. Chang, On the characterizations of the breakdown points of quasilinear wave equations..............................

Joseph Nicholas Fadyn, The projectivity of $\operatorname{Ext}(T, A)$ as a module over

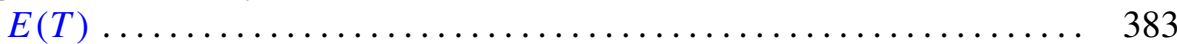

Donald Eugene Maurer, Arithmetic properties of the idèle discriminant .... 393

Stuart Rankin, Clive Reis and Gabriel Thierrin, Right subdirectly irreducible semigroups ................................. 403

David Lee Rector, Homotopy theory of rigid profinite spaces. I ........ 413 Raymond Moos Redheffer and Wolfgang V. Walter, Comparison theorems for parabolic functional inequalities................

H. M. (Hari Mohan) Srivastava, Some generalizations of Carlitz's theorem

James Alan Wood, Unbounded multipliers on commutative Banach algebras....

T. Yoshimoto, Vector-valued ergodic theorems for operators satisfying norm

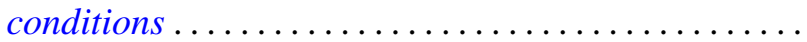

Jerry Searcy and B. Andreas Troesch, Correction to: "A cyclic inequality and a related eigenvalue problem".

Leslie Wilson, Corrections to: "Nonopenness of the set of Thom-Boardman maps" 